\title{
Non-Equilibrium Dynamics of Two-dimensional Infinite Particle Systems with a Singular Interaction
}

\section{J. Fritz}

Mathematical Institute, Budapest, Hungary

\section{R. L. Dobrushin}

Institute for Problems of Information Transmission, Moscow, USSR

\begin{abstract}
The infinite system of Newton's equations of motion is considered for two-dimensional classical particles interacting by conservative two-body forces of finite range. Existence and uniqueness of solutions is proved for initial configurations with a logarithmic order of energy fluctuation at infinity. The semigroup of motion is also constructed and its continuity properties are discussed. The repulsive nature of interparticle forces is essentially exploited; the main condition on the interaction potential is that it is either positive or has a singularity at zero interparticle distance, which is as strong as that of an inverse fourth power.
\end{abstract}

\section{Introduction}

In this paper we extend some of our earlier results [3] on the existence of nonequilibrium dynamics of one-dimensional infinite particle systems to infinite systems of two-dimensional particles interacting by conservative repulsive forces of finite range. For a detailed motivation of this problem see [1-3], where further references are given on equilibrium dynamics as well.

Consider a finite or infinite system $\omega$ of two-dimensional particles. We assume that particles are numbered by a nonempty subset $J$ of the set $I$ of integers, the position and the velocity of the $i$-th particle, $i \in J$, will be denoted by $x_{i}$ and $v_{i}$, respectively. Conservative two-body forces are given by the negative gradient $F=-\operatorname{grad} U$ of a symmetric real function $U=U(x)$ of two variables $\left(x^{(1)}, x^{(2)}\right)=x$, $U$ is the interaction potential. For equal particles of unit mass indexed by $J \subset I$, Newton's equations of motion read formally as

$$
\frac{d v_{i}}{d t}=-\sum_{j \in J_{i}} \operatorname{grad} U\left(x_{i}-x_{j}\right), \quad \frac{d x_{i}}{d t}=v_{i} ; \quad i \in J
$$

with initial conditions specifying the position and the velocity of each particle at time zero. The full system, when $J=I$, will be denoted as (NI), $J_{i}=\{j ; j \in J, j \neq i\}$ if $i \in J$. 
Such a set $\boldsymbol{\Omega}_{0}$ of configurations will be defined in an explicit way that for each initial condition $\omega_{0} \in \boldsymbol{\Omega}_{0}$ the corresponding sequence of solutions to finite subsystems (NJ) converges in the weak sense to a solution to the infinite system (NI); i.e. for $\omega_{0} \in \boldsymbol{\Omega}_{0}$ there exists a limiting solution with initial configuration $\omega_{0}$. The set $\boldsymbol{\Omega}_{0}$ is characterized by a logarithmic order of energy fluctuation at infinity, it is of full measure with respect to a wide class of Gibbsian fields with superstable interactions. Limiting solutions form a reversible semigroup of motion in $\Omega_{0}$. Uniqueness and continuous dependence of solutions on initial data also hold in a restricted sense. For the proof of existence of limiting solutions the method of [3] has been developed further.

Regularity conditions on the interaction potential $U=U(x)$ are somewhat stronger than in the one-dimensional case [3]. Hard-core interactions are not allowed, and for not necessarily nonnegative potentials we need such an assumption that the singularity of $U$ at $x=0$ is of type $|x|^{-b}$ with $b \geqq 4$. It is not very difficult to understand on a physical level that why are such conditions necessary to avoid a breakdown of solutions with good initial conditions. If the interaction is very singular (e.g. as in the presence of hard cores) then the velocity of the propagation of shock waves along linear chains of particles can be arbitrarily large, so that large energy can be transferred to the center in a short time along a radial system of chains. On the other hand, if the repulsive nature of the interaction is not strong enough, then too many particles can be accumulated in a small region, and one of them may win almost the total energy of two or more others. These phenomena are typical in two or more dimensions, and as shown by J. Ginibre in the case of hard cores (unpublished result, personal communication by D. Ruelle) they can result in a breakdown of solutions even if the initial velocities are uniformly bounded.

As indicated in Section 4 of [3], our methods do not work in the threedimensional case. Let us present a heuristic example suggesting that breakdown of solutions may be typical in three dimensions even for potentials without a hard core. At time zero, particles are sitting in the points of the integer lattice with approximately equal magnitude of velocities, each second cubic cell of the lattice is red. Imagine now that particles from the vertices of each red cube are directed towards the center of the cube, and after the collision seven of them remains at rest while the eighth one flies away in such a direction that the initial situation will be repeated on the new lattice formed by the centers of red cubes, and so on. Let $v_{n}$ denote the approximate magnitude of velocities of flying particles after the $n$-th collision; the conservation laws for energy and inpulse allow that $v_{n+1} \geqq v_{n} \varrho$ with $\varrho>2$. Since the $n+1$-thlattice is only twice as large as the $n$-th one, infinite velocities appear in a finite time. Unfortunatelly we are not able to carry out this construction in a rigorous way.

\section{Notation}

$\boldsymbol{R}^{2}$ denotes the two-dimensional Euclidean space with the usual norm $|x|$ and scalar product $(x, y)$. The set of locally finite labelled configurations is $\boldsymbol{\Omega}$, a configuration $\omega \in \boldsymbol{\Omega}$ is a finite or countable sequence of pairs of positions $x_{i} \in \boldsymbol{R}^{2}$ and velocities $v_{i} \in \boldsymbol{R}^{2}$; i.e. configurations are represented as $\omega=\left\{\left\{x_{i}, v_{i}\right\} ; i \in J\right\}$, where the index set 
$J=J(\omega)$ is a nonempty subset of the set of integers $I, x_{i} \neq x_{j}$ if $i \neq j$, and the sequence $\left\{x_{i}\right\}, i \in J$ has no limit points. The position and the velocity of the $i$-th particle in $\omega$ will be denoted as $x_{i}=x_{i}(\omega), v_{i}=v_{i}(\omega)$, respectively. Configurations differing only in the way of enumeration of particles are usually identified, but the equations of motion and the statements are formulated in terms of labelled configurations.

In the space of subsets of $I$ we consider the discrete topology, i.e. $\lim J_{n}=J$ means that for each finite $V \subset I$ there exists an $n_{V}$ such that $n>n_{V}$ implies that $V \cap J_{n}=V \cap J$. The configuration space $\Omega$ is equipped with the weak topology, i.e. $\lim _{n} \omega_{n}=\omega$ in $\boldsymbol{\Omega}$ means that $J(\omega)=\lim _{n} J\left(\omega_{n}\right)$ and $x_{i}(\omega)=\lim _{n} x_{i}\left(\omega_{n}\right), v_{i}(\omega)=\lim _{n} v_{i}\left(\omega_{n}\right)$ for each $i \in J(\omega)$ without any uniformity condition in $i$. It is easy to check that this convergence relation corresponds to a metrizable topology. Trajectories in $\boldsymbol{\Omega}$ are parametrized by the time $t \geqq 0$, the set of weakly continuous trajectories $\omega_{t}=\varphi(t)$, $\omega_{t} \in \boldsymbol{\Omega}$ for $t \geqq 0$ will be denoted by $\boldsymbol{\Omega}[0, \infty)$. Observe that $J\left(\omega_{t}\right)$ does not depend on $t$ if $\omega_{t} \in \boldsymbol{\Omega}[0, \infty)$ and particles along a continuous trajectory preserve their initial numbering. Convergence $\lim _{n} \omega_{t}^{n}=\omega_{t}$ in $\boldsymbol{\Omega}[0, \infty)$ means that $\lim _{n} \omega_{t}^{n}=\omega_{t}$ for each $t \geqq 0$ and this weak convergence is uniform in finite intervals of time. The underlying topology of $\boldsymbol{\Omega}[0, \infty)$ is metrizable, too.

A family $\omega_{t}=\varphi(t, \omega), \omega \in \boldsymbol{\Omega}^{\prime}$ is a reversible semigroup in $\boldsymbol{\Omega}^{\prime} \subset \boldsymbol{\Omega}$ if $\omega_{t} \in \boldsymbol{\Omega}^{\prime}$ for $t \geqq 0$, further $\varphi(0, \omega)=\omega, \varphi(t+s, \omega)=\varphi(t, \varphi(s, \omega))$ and $\varphi\left(t,[\varphi(t, \omega)]^{+}\right)=\omega^{+}$are identities ; in the last one $\omega^{+}$is defined by $x_{i}\left(\omega^{+}\right)=x_{i}(\omega), v_{i}\left(\omega^{+}\right)=-v_{i}(\omega), i \in J(\omega)=J\left(\omega^{+}\right)$.

Consider now a translation invariant pair potential $U$ of range $R>0$. To avoid such situations when two or more particles can be found at the same point of $\boldsymbol{R}^{2}$, we assume that $U$ is singular and repulsive. Then $U$ is given by a continuously differentiable real function $U=U(x), x \in \boldsymbol{R}^{2}, x \neq 0$ such that $U(x)=U(-x), U(x)=0$ if $|x| \geqq R$ and $\lim _{x \rightarrow 0} U(x)=+\infty$. For convenience we assume that the interparticle force $F=-\operatorname{grad} U(x)$ satisfies a local Lipschitz condition at each $x \neq 0$, then the best constant $L=L(u), u \geqq 0$ such that

$$
|\operatorname{grad} U(x)-\operatorname{grad} U(y)| \leqq L(u)|x-y|
$$

if $U(x) \leqq u, U(y) \leqq u$, is finite for each $u$.

The number of particles and the total energy are set functions for each configuration $\omega \in \boldsymbol{\Omega}$. Let $f_{0}(x, \sigma)=1$ if $|x| \leqq \sigma$ and $f_{0}=0$ otherwise, further $J=J(\omega)$, $x_{i}=x_{i}(\omega), v_{i}=v_{i}(\omega)$, then

$$
N(\omega, \mu, \sigma)=\sum_{i \in J} f_{0}\left(x_{i}-\mu, \sigma\right)
$$

and

$$
H(\omega, \mu, \sigma)=\sum_{i \in J} f_{0}\left(x_{i}-\mu, \sigma\right) \frac{1}{2}\left[\left|v_{i}\right|^{2}+\sum_{j \in J_{i}} f_{0}\left(x_{j}-\mu, \sigma\right) U\left(x_{i}-x_{j}\right)\right]
$$

are the number and the total energy of particles of $\omega$ in the $\sigma$-neighborhood of $\mu \in \boldsymbol{R}^{2}$.

A weakly continuous trajectory $\omega_{t} \in \boldsymbol{\Omega}[0, \infty)$ is called a (global) solution to the equations of motion with initial condition $\omega \in \boldsymbol{\Omega}$ if $\omega_{0}=\omega$, the individual 
trajectories $x_{i}=x_{i}\left(\omega_{t}\right), v_{i}=v_{i}\left(\omega_{t}\right)$ are differentiable and satisfy (NJ) with $J=J\left(\omega_{0}\right)$ for $t \geqq 0$. If $J$ is finite then the law of energy conservation and $L(u)<\infty$ imply the existence of a unique solution to (NJ). Set

$$
J^{n}(\omega)=\left\{j ;\left|x_{j}(\omega)\right| \leqq n, j \in J(\omega)\right\}
$$

for each $\omega \in \boldsymbol{\Omega}$, and let $\omega_{t}=\varphi_{n}(t, \omega)$ denote the unique solution to $\left(\mathrm{NJ}^{n}(\omega)\right)$ with initial condition $x_{i}\left(\omega_{0}\right)=x_{i}(\omega), v_{i}\left(\omega_{0}\right)=v_{i}(\omega)$ for $i \in J^{n}(\omega)$. A solution $\omega_{t}$ is called a limiting solution to the equations of motion if we can select a subsequence $n_{k}$ such that $\omega_{t}=\lim _{k} \varphi_{n_{k}}\left(t, \omega_{0}\right)$ in the topology of $\boldsymbol{\Omega}[0, \infty)$.

\section{Main Result}

The allowed set of initial configurations and the boundary condition needed for uniqueness of solutions are defined in terms of the order of energy fluctuation at infinity that is given by an SI-function $g$.

Definition 1. An increasing concave function $g=g(u), u \geqq 0$ is called an SI-function if $g(0)=1, g(u) \leqq 1+2 \sqrt{u}, g^{\prime}(0+0)=1$ and $\int_{1}^{\infty}[u g(u)]^{-1} d u=\infty ; g^{\prime}(u+0)$ denotes the right derivative of $g$ at $u$. We say that energy fluctuation of $\omega \in \boldsymbol{\Omega}$ is only of order $g$ if

$$
\bar{H}_{g}(\omega)=\sup _{\mu} \sup _{\sigma \geqq g(|\mu|)} \sigma^{-2} H(\omega, \mu, \sigma)
$$

is finite, the set of such configurations will be denoted by $\boldsymbol{\Omega}_{g}$.

The particular case $g_{0}(u)=1+\log (1+u)$ is of special interest, the corresponding set of configurations will be denoted by $\boldsymbol{\Omega}_{0} ; \log u$ denotes logarithm to base $e$, and $\overline{\boldsymbol{\Omega}}=\cup \boldsymbol{\Omega}_{g}$, where the union is over all SI-functions. Limiting solutions will be constructed for initial configurations belonging to $\overline{\boldsymbol{\Omega}}$, but even $\boldsymbol{\Omega}_{0}$ carries a wide class of probability measures.

Let $V=V\left(x_{1}, x_{2}, \ldots, x_{n}\right), \quad x_{i} \in \boldsymbol{R}^{2}, n=2,3, \ldots$ denote a translation invariant multibody interaction (see Section 1 in [4]). A probability measure $\boldsymbol{P}$ on the Borel subsets of $\boldsymbol{\Omega}$ is called a Gibbsian field (of activity $z$ and inverse temperature $\beta$ ) with interaction $V$ if the conditional distribution of the positions of particles in each bounded domain is given by the corresponding conditional grand canonic Gibbs distribution, further the velocity co-ordinates are independent Gaussian variables of mean 0 and variance $\beta^{-1}$.

Proposition 1. Suppose that $\boldsymbol{P}$ is a Gibbsian field with interaction $V=V_{1}+V_{2}$, where $V_{1}$ corresponds to a superstable and lower regular pair potential $U_{1}=U_{1}(x), x \in \boldsymbol{R}^{2}, V_{2}$ is a stable and lower regular multibody interaction (see Section 1 in [4]). If $U(x) \leqq a_{1}+b_{1} U_{1}(x)$ with some constants $a_{1}$ and $b_{1}$, then $\boldsymbol{P}\left(\boldsymbol{\Omega}_{0}\right)=1$.

The proof of this statement is essentially the same as that of Proposition 1 in [3]. Using Ruelle's superstability estimate [4], a direct calculation results in

$$
\int \exp (\lambda H(\omega, \mu, \sigma)) \boldsymbol{P}(d \omega) \leqq e^{K \sigma^{2}}
$$

with some $\lambda>0$ and $K<+\infty$, whence $\boldsymbol{P}\left(\boldsymbol{\Omega}_{0}\right)=1$ follows by the Markov inequality and the Borel-Cantelli lemma. 
To prove existence of limiting solutions we need the following regularity conditions on the interaction potential $U$ : There exist such positive constants $a, b, c$, and $\delta$ that

$$
|x| \operatorname{|grad} U(x) \mid \leqq a+b U(x),
$$

further $U(x)>0$ if $|x| \leqq \delta$, and either

$$
|\operatorname{grad} U(x)|^{2} \leqq c U(x) \quad \text { if } \quad|x| \geqq \delta,
$$

or

$$
c U(x) \geqq|x|^{-4} \quad \text { if } \quad|x| \leqq \delta
$$

holds. Let us remark that $(\mathrm{E})$ means that the singularity of $U$ at 0 is not stronger than that of $|x|^{-b}$, and $U(x) \geqq-\frac{a}{b}$ also follows from (E). If $U$ is twice continuously differentiable for $x \neq 0$ and $U \geqq 0$, then (P) always holds. Further, any of $(\mathrm{P})$ and $(\mathrm{R})$ implies that $U$ is a superstable potential. The validity of $(\mathrm{E})$ and one of $(\mathrm{P})$ and $(\mathrm{R})$ will be assumed throughout this paper. Our main result is

Theorem 1. For each $\omega \in \overline{\boldsymbol{\Omega}}$ there exists a limiting solution $\omega_{t}$ with initial condition $\omega_{0}=\omega$.

The study of not necessarily limiting solutions is based on the following regularity property.

Definition 2. We say that a solution $\omega_{t}$ is tempered if it is $g$-tempered with some SIfunction $g ; g$-temperedness of $\omega_{t}$ means that $\bar{H}_{g}\left(\omega_{t}\right)$ is bounded in finite intervals of time.

Theorem 2. Any limiting solution $\omega_{t}$ is tempered if $\omega_{0} \in \overline{\boldsymbol{\Omega}}$, and a tempered solution $\bar{\omega}_{t}$ is $g$-tempered with an SI-function $g$ if and only if $\bar{\omega}_{t} \in \boldsymbol{\Omega}_{g}$ for at least one value of $t \geqq 0$.

Uniqueness of tempered solutions can be proved under the following quasiLipschitz condition.

Definition 3. We say that $U$ satisfies a $g$-Lipschitz condition with an SI-function $g$ if

$$
\lim _{u \rightarrow \infty} \sqrt{u}\left[g^{-1}(v \sqrt{u})\right]^{-2} L(u)=0 \text { for each } v>0,
$$

where $L$ is defined in (1), $g^{-1}$ denotes the inverse function of $g$.

(U) means that the singularity of $U$ is not very weak. Indeed, if $U$ satisfies (R) with an exponent $\lambda<0$ instead of -4 , further $U$ is rotation invariant and $|\operatorname{grad} U(x)|$ is a convex function of $r=|x|$ in a neighborhood of 0 , then (U) holds with any SIfunction $g$.

Theorem 3. Suppose that $U$ satisfies a g-Lipschitz condition with an SI-function $g$. Then for each $\omega \in \boldsymbol{\Omega}_{g}$ there exists the limit $\boldsymbol{U}_{t} \omega=\lim _{n} \varphi_{n}(t, \omega)$ of approximate solutions. $\boldsymbol{U}_{t}$ is a reversible semigroup of g-tempered solutions, and $\omega_{t}=\boldsymbol{U}_{t} \omega_{0}$ is the only tempered solution with initial configuration $\omega_{0} \in \boldsymbol{\Omega}_{g}$. Moreover, the restriction of $\boldsymbol{U}_{t}: \boldsymbol{\Omega}_{g} \mapsto \boldsymbol{\Omega}[0, \infty)$ to $\boldsymbol{\Omega}_{g}^{h}=\left\{\omega ; \omega \in \boldsymbol{\Omega}, \bar{H}_{g}(\omega) \leqq h\right\}$ is a continuous function of $\omega \in \boldsymbol{\Omega}_{g}^{h}$ for each $h<\infty$. 
The theorems will be proven in Sections 4-6; now we outline the main steps of the proofs. The basic result is the following compactness property of $g$-tempered solutions. For each SI-function $g$ and positive number $h<\infty$ there exists a continuous function $q(t)=q(t, h), t \geqq 0$ such that $\bar{H}_{g}\left(\omega_{t}\right) \leqq q(t, h)$ for each $t \geqq 0$ whenever $\omega_{t}$ is a $g$-tempered solution with $\omega_{0} \in \boldsymbol{\Omega}_{g}^{h}$. This a priori bound implies that $g$-tempered solutions $\omega_{t}, \omega_{0} \in \boldsymbol{\Omega}_{g}^{h}$ form a compact subset of $\boldsymbol{\Omega}[0, \infty)$ including solutions to the finite systems (NJ). The a priori bound $\bar{H}_{g}\left(\omega_{t}\right) \leqq h(t)$ can be actually proved for such a nonnegative and smooth version $W=W(\omega, \mu, \sigma)$ of $H$ that $H(\omega, \mu, \sigma) \leqq W(\omega, \mu, \sigma)$ and

$$
\bar{W}_{g}(\omega)=\sup _{\mu} \sup _{\sigma \geqq g(|\mu|)} \sigma^{-2} W(\omega, \mu, \sigma)
$$

is bounded by a linear function of $\bar{H}_{g}(\omega)$. This $W$ will be introduced in the next section where we show also that $W$ satisfies the partial differential inequality

$$
\begin{aligned}
\frac{\partial}{\partial t} W\left(\omega_{t}, \mu, \sigma\right) \leqq & K W\left(\omega_{t}, \mu, \sigma\right) \\
& +K g(|\mu|+\sigma) \sqrt{W_{g}\left(\omega_{t}\right)} \frac{\partial}{\partial \sigma} W\left(\omega_{t}, \mu, \sigma\right)
\end{aligned}
$$

along any solution $\omega_{t} \in \boldsymbol{\Omega}_{g} ; K$ is a constant depending only on $U, g$ is an SI-function. For a heuristic motivation of the role played by $W$ see Section 4 in [3]. In a similar way as in [3], (6) reduces to the differential inequality

$$
z^{\prime}(t) \leqq \bar{K} \exp \left(\frac{1}{2} K t\right) \bar{W}_{g}\left(\omega_{0}\right)\left[1+z g\left(z^{2}\right)\right]
$$

for $z(t)=\int_{0}^{t}\left(\bar{W}_{g}\left(\omega_{s}\right)\right)^{1 / 2} d s ; \bar{K}$ is a new constant depending only on $K$. Since $g$ is an SIfunction, (7) has a continuous maximal solution defined for $t \geqq 0$, therefore $\bar{W}_{g}\left(\omega_{t}\right)$ is bounded by the very same continuous function $\varrho(t)=\varrho(t, w)$ for each $g$-tempered solution $\omega_{t}$ satisfying $\bar{W}_{g}\left(\omega_{0}\right) \leqq w$. Hence existence of limiting solutions follows by the standard compactness argument. Uniqueness of tempered solutions is implied by a contraction property of the right hand side of $(\mathrm{NJ})$ due to (U).

\section{Proof of (6)}

Let $f=f(u)$ denote such a continuously differentiable nondecreasing function that

(i) $f(u)=0$ if $u \leqq-3 R, f(u)=1$ if $u \geqq 0, f\left(-\frac{5}{2} R\right)=\frac{1}{3}, f\left(-\frac{R}{2}\right)=\frac{2}{3}$.

(ii) $f$ is convex for $u \leqq-\frac{R}{2}$ and it is concave for $u \geqq-\frac{5}{2} R$, i.e. $f$ is linear if $-\frac{5}{2} R \leqq u \leqq-\frac{R}{2}$.

(iii) There exists a constant $d>0$ such that $\left|f^{\prime}(u)\right|^{2} \leqq d f(u)$.

Then the derivative $f^{\prime}$ of $f$ is nonnegative and (ii) implies that

(iv) $f^{\prime}(u) \leqq f^{\prime}(v)+f^{\prime}(z)$ if $v \leqq u \leqq z \leqq v+2 R$. 
If $\omega \in \boldsymbol{\Omega}, \mu \in \boldsymbol{R}^{2}, \sigma \geqq 1$ and $x_{i}=x_{i}(\omega), v_{i}=v_{i}(\omega), J=J(\omega)$, then $W$ is defined as

$$
W(\omega, \mu, \sigma)=\sum_{i \in J} f\left(\sigma-\left|x_{i}-\mu\right|\right) W_{i}(\omega),
$$

where

$$
W_{i}(\omega)=1+\left|v_{i}\right|^{2}+\sum_{j \in J_{i}}\left[\lambda f\left(3 R-3\left|x_{i}-x_{j}\right|\right)+U\left(x_{i}-x_{j}\right)\right]
$$

and $\lambda=\frac{a}{b}$ if $(\mathrm{R})$ holds, $\lambda=0$ otherwise. Let us remark that $U(x) \geqq-\frac{a}{b}$ in view of $(\mathrm{E})$, thus $W_{i}(\omega) \geqq 1$ and $W$ is a nondecreasing function of $\sigma$. Consider now the quantity $\bar{W}_{g}(\omega)$ defined in (5) by (8) and (9), $g$ is an SI-function. It is plain that

$$
\left|v_{i}\right| \leqq A g(|\mu|+\sigma)\left[\bar{W}_{g}(\omega)\right]^{1 / 2} \quad \text { if } \quad\left|x_{i}-\mu\right| \leqq \sigma+5 R
$$

and $A \geqq 1+5 R$, further $U_{+}(x)=\lambda f(3 R-3|x|)+U(x) \geqq 0$ is a superstable pair potential, thus the constant $A$ can be choosen to be so large that even

$$
N_{i} \leqq A g(|\mu|+\sigma)\left[\bar{W}_{g}(\omega)\right]^{1 / 2} \text { if }\left|x_{i}-\mu\right| \leqq \sigma+3 R,
$$

where $N_{i}=N\left(\omega, x_{i}, 2 R\right)-1 ;(11)$ is a direct consequence of the definition of superstability (see [4], [5] or [7]) and of $W_{i}(\omega) \geqq 1$.

Now we are in a position to prove (6). Let $\omega_{t}$ denote any solution to the equations of motion such that $\omega_{t} \in \boldsymbol{\Omega}_{g}$ for each $t \geqq 0$, then $W\left(\omega_{t}, \mu, \sigma\right)$ is a differentiable function of $t$. For notational convenience the following abbreviations will be used: $e_{i}$ and $e_{i j}$ are the unit vector directed as $\mu-x_{i}$ and $x_{j}-x_{i}$, while $f_{i}, f_{i}^{\prime}$, $f_{i j}, f_{i j}^{\prime}$ denote the value of $f$ and of $f^{\prime}$ at $\sigma-\left|\mathrm{x}_{i}-\mu\right|$ and $3 R-3\left|x_{i}-x_{j}\right|$, respectively. Differentiating $W\left(\omega_{t}, \mu, \sigma\right)$ with respect to $t$ and exploiting $\operatorname{grad} U\left(x_{i}-x_{j}\right)+\operatorname{grad} U\left(x_{j}-x_{i}\right)=0$ and the equations of motion, an easy calculation yields

$$
\frac{\partial}{\partial t} W\left(\omega_{t}, \mu, \sigma\right)=Q_{1}+Q_{2}+Q_{3},
$$

where

$$
\begin{aligned}
& Q_{1}=\sum_{i \in J} f_{i}^{\prime}\left(e_{i}, v_{i}\right) W_{i}\left(\omega_{t}\right), \\
& Q_{2}=\frac{1}{2} \sum_{i \in J} \sum_{j \in J_{i}}\left(f_{j}-f_{i}\right)\left(\operatorname{grad} U\left(x_{i}-x_{j}\right), v_{i}+v_{j}\right), \\
& Q_{3}=\sum_{i \in J} f_{i} \sum_{j \in J_{i}} 3 \lambda f_{i j}^{\prime}\left(e_{i j}, v_{i}-v_{j}\right) .
\end{aligned}
$$

These sums are estimated as follows. Since

$$
\begin{aligned}
& \frac{\partial}{\partial \sigma} W\left(\omega_{t}, \mu, \sigma\right)=\sum_{i \in J} f_{i}^{\prime} W_{i}\left(\omega_{t}\right), \\
& Q_{1} \leqq A V \frac{\partial}{\partial \sigma} W\left(\omega_{t}, \mu, \sigma\right),
\end{aligned}
$$

where

$$
V=V_{g}\left(\omega_{t}, \mu, \sigma\right)=g(|\mu|+\sigma)\left[\bar{W}_{g}\left(\omega_{t}\right)\right]^{1 / 2}
$$

denotes the nonconstant factor in (10). 
Applying the Lagrange theorem and (iv) for $f_{j}-f_{i}$, it follows from (E) and (P) that

$$
\begin{aligned}
Q_{2} & \leqq \frac{1}{2} \sum_{i \in I} \sum_{j \in J_{i}}\left(f_{i}^{\prime}+f_{j}^{\prime}\right)\left|x_{i}-x_{j}\right|\left|\operatorname{grad} U\left(x_{i}-x_{j}\right)\right|\left|v_{i}+v_{j}\right| \\
& \leqq \sum_{i \in J} f_{i}^{\prime} \sum_{j \in J_{i}}\left[\bar{f}_{i j}+b U\left(x_{i}-x_{j}\right)\right]\left(\left|v_{i}\right|+\left|v_{j}\right|\right),
\end{aligned}
$$

where $\bar{f}_{i j}=a f_{i j}$ if $(\mathrm{P})$ does not hold, i.e. $\lambda=\frac{a}{b}$ in (9); otherwise $\bar{f}_{i j}$ is defined as $\bar{f}_{i j}=a$ if $\left|x_{i}-x_{j}\right| \leqq \delta, \bar{f}_{i j}=R\left[c U\left(x_{i}-x_{j}\right)\right]^{1 / 2}$ if $\left|x_{i}-x_{j}\right|>\delta$. In the first case we have

$$
\begin{aligned}
Q_{2} & \leqq 2 A V \sum_{i \in J} f_{i}^{\prime} \sum_{j \in J_{i}}\left[a f_{i j}+b U\left(x_{i}-x_{j}\right)\right] \\
& \leqq 2 b A V \frac{\partial}{\partial \sigma} W\left(\omega_{t}, \mu, \sigma\right) .
\end{aligned}
$$

On the other hand, if (P) holds then there exist such constants $K_{1}$ and $K_{2}$ depending only on $U$ that $\bar{f}_{i j} \leqq K_{1}$ and $\bar{f}_{i j} \leqq K_{2}\left[U\left(x_{i}-x_{j}\right)\right]^{1 / 2}$, thus in this case we obtain

$$
Q_{2} \leqq 2 b A V \frac{\partial}{\partial \sigma} W\left(\omega_{t}, \mu, \sigma\right)+K_{1} S_{1}+K_{2} S_{2}
$$

where

$$
S_{1}=\sum_{i \in J} f_{i}^{\prime}\left|v_{i}\right| N_{i} \leqq A V \frac{\partial}{\partial \sigma} W\left(\omega_{t}, \mu, \sigma\right)
$$

in view of (11) and of $\left|v_{i}\right| \leqq 1+\left|v_{i}\right|^{2}$, further

$$
S_{2}=\sum_{i \in J} f_{i}^{\prime} \sum_{j \in J_{i}}\left|v_{j}\right|\left|U\left(x_{i}-x_{j}\right)\right|^{1 / 2}
$$

Set $J_{i}^{\prime}=\left\{j ;\left|x_{i}-x_{j}\right| \leqq 2 R, j \in J_{i}\right\}$, then

$$
\left[\sum_{j \in J_{i}^{\prime}}\left|v_{j}\right|^{2}\right]^{1 / 2} \leqq A V
$$

holds with the same constant $A$ as (10) does, therefore applying the Cauchy inequality to the second sum in (23) we obtain that

$$
\begin{aligned}
S_{2} & \leqq \sum_{i \in J} f_{i}^{\prime} A V\left[\sum_{j \in J^{\prime}} U\left(x_{i}-x_{j}\right)\right]^{1 / 2} \\
& \leqq A V \sum_{i \in J} f_{i}^{\prime}\left[1+\sum_{j \in J_{i}} U\left(x_{i}-x_{j}\right)\right] \leqq A V \frac{\partial}{\partial \sigma} W\left(\omega_{t}, \mu, \sigma\right)
\end{aligned}
$$

Observe now that $f_{i j}^{\prime}=0$ if $\left|x_{i}-x_{j}\right| \geqq 2 R$, thus $Q_{3}$ can be splitted into the following three terms :

$$
Q_{3}=6 \lambda Q_{31}+6 \lambda Q_{32}+3 \lambda Q_{33},
$$


where

$$
\begin{aligned}
& Q_{31}=\sum_{i \in J} f_{i} \sum_{j \in J_{i}^{\prime}} f_{j} f_{i j}^{\prime}\left(e_{i j}, v_{i}\right), \\
& Q_{32}=\sum_{i \in J} f_{i} \sum_{j \in J_{i}^{\prime}}\left(1-f_{j}\right) f_{i j}^{\prime}\left(e_{i j}, v_{i}\right)
\end{aligned}
$$

and

$$
\begin{aligned}
Q_{33} & =-\sum_{i \in J} f_{i} \sum_{j \in J_{i}^{\prime}} f_{i j}^{\prime}\left(e_{i j}, v_{i}+v_{j}\right) \\
& =\frac{1}{2} \sum_{i \in J} \sum_{j \in J_{i}^{\prime}}\left(f_{j}-f_{i}\right) f_{i j}^{\prime}\left(e_{i j}, v_{i}+v_{j}\right) \\
& \leqq 2 R \sum_{i \in J} f_{i}^{\prime} \sum_{j \in J_{i}^{\prime}} f_{i j}^{\prime}\left|v_{i}+v_{j}\right|
\end{aligned}
$$

by the Lagrange Theorem and (iv). Let

$$
\begin{aligned}
& S_{3}=\sum_{i \in J} f_{i}^{\prime} \sum_{j \in J_{i}^{\prime}} f_{i j}^{\prime}\left|v_{j}\right|, \\
& S_{4}=\sum_{i \in J} f_{i}\left|v_{i}\right| \sum_{j \in J_{i}^{\prime}} f_{j}
\end{aligned}
$$

since $f_{i}>\frac{2}{3}$ and $f_{i j}^{\prime} \neq 0$ imply that $f_{j} \geqq \frac{1}{3}$, we have

$$
\left(1-f_{j}\right) f_{i j}^{\prime} \leqq 2 f_{j} f_{i j}^{\prime} \leqq(2+2 d) f_{j} \quad \text { if } \quad f_{i}>\frac{2}{3}
$$

as $f_{i j}^{\prime} \leqq 1+d$ in view of (ii) and (iii). On the other hand, the Lagrange theorem and (i) and (iv) imply that

$$
f_{i}=f_{i}-f(-3 R) \leqq 3 R f_{i}^{\prime} \quad \text { if } f_{i} \leqq \frac{2}{3},
$$

consequently

$$
Q_{32} \leqq 3 R(1+d) S_{2}+(2+2 d) S_{4} .
$$

Consider now the sums $Q_{31}$ and $Q_{33}$. Since $f_{i j}^{\prime} \leqq 1+d$, we have

$$
Q_{31} \leqq(1+d) S_{4},
$$

further

$$
Q_{33} \leqq(2+2 d) R S_{1}+2 R S_{3}
$$

but

$$
S_{3} \leqq(1+d) A V \frac{\partial}{\partial \sigma} W\left(\omega_{t}, \mu, \sigma\right)
$$

follows in the same way from (iii) and (24) as (25) has been derived.

To this point we have shown that $\frac{\partial W}{\partial t}$ is bounded by a linear combination of $S_{1}$, $S_{2}, S_{3}$, and of $S_{4}$. In view of (22), (25), and of (37), the last step is to bound $S_{4}$ by a constant multiple of $W\left(\omega_{t}, \mu, \sigma\right)$. For this the following elementary inequality is needed. 
Lemma. Let $V_{\delta}(y)$ denote the square of center $y \in \boldsymbol{R}^{2}$ and diameter $\delta>0$. The sides of $V_{\delta}(y)$ are parallel to the co-ordinate axes, $J(y)=\left\{i ; x_{i} \in V_{\delta}(y)\right\}, J_{i}(y)=J \cap J_{i}$; then whenever $J(y)$ has at least two elements, we have

$$
\left[\sum_{i \in J(y)} f_{i}\right]^{3} \leqq \bar{c} \sum_{i \in J(y)} f_{i} \sum_{j \in J_{i}(y)}\left|x_{i}-x_{j}\right|^{-4},
$$

where the constant $\bar{c}$ is independent of the underlying configuration $\omega=\omega_{t}$.

Proof. Let $J_{\varepsilon}$ denote the set of such $i \in J(y)$ that $\left|x_{i}-x_{j}\right| \leqq \varepsilon$ holds at least for one $j \in J_{i}(y)$, and set

$$
\Sigma=\sum_{i \in J(y)} f_{i}, \quad \Sigma_{\varepsilon}=\sum_{i \in J_{\varepsilon}} f_{i} .
$$

We assume that $0<\varepsilon<\delta$, then the $\frac{\varepsilon}{2}$-neighborhoods of such points $x_{k}$ that $k \notin J_{\varepsilon}$ are disjoint and each of them is contained in the same square of side $2 \delta$ and center $y$, thus

$$
\frac{3}{4} \varepsilon^{2}\left(\Sigma-\Sigma_{\varepsilon}\right) \leqq 4 \delta^{2} .
$$

On the other hand,

$$
\varepsilon^{-4} \Sigma_{\varepsilon} \leqq P
$$

where $P$ denotes the sum on the right of (38), i.e. $\bar{c} P$ dominates the left hand side of (38).

First we assume that $\Sigma>9$, then $\varepsilon=3 \delta \Sigma^{-1 / 2}<\delta$ and (39) results in $\Sigma \leqq 3 \Sigma_{\varepsilon}$, whence by (40) we obtain

$$
\Sigma^{3} \leqq 3^{5} \delta^{4} P
$$

In the second case we may assume that $3^{5} \delta^{4} P \leqq 3^{6}$, i.e. $P \leqq 3 \delta^{-4}$. Therefore the trivial bound $\delta^{-4} \Sigma \leqq P$ implies $\Sigma^{3} \leqq 9 \delta^{-20} P$, thus (38) holds with $\bar{c}=\max \left\{3^{5} \delta^{4}, 9 \delta^{-20}\right\}$.

Now we can estimate $S_{4}$, too. Observe first that

$$
S_{4} \leqq \sum_{i \in J} f_{i}\left|v_{i}\right|^{2}+\sum_{i \in J} f_{i}\left[\sum_{j \in J_{i}^{\prime}} f_{j}\right]^{2},
$$

and the first sum on the right is not larger than the actual value of $W\left(\omega_{t}, \mu, \sigma\right)$. On the other hand, (R), (38) and the relation $W_{i}\left(\omega_{t}\right) \geqq 1$ imply that

$$
\left[\sum_{i \in J(y)} f_{i}\right]^{3} \leqq\left(1+\frac{\bar{c}}{c}\right) \sum_{i \in J(y)} f_{i} W_{i}\left(\omega_{t}\right)
$$

holds even if the cardinality of $J(y)$ is less than two. Let $Z^{2}$ denote the points of the integer lattice in $\boldsymbol{R}^{2}, \boldsymbol{Z}_{\delta}^{2}=\left\{y ; \sqrt{2} \delta^{-1} y \in \boldsymbol{Z}^{2}\right\}, A_{y}=\left\{x ; x \in \boldsymbol{Z}_{\delta}^{2},|x-y| \leqq 2 R+\delta\right\}$ and $k$ is the cardinality of $A_{y}$. Then for $y \in Z_{\delta}^{2}$ we have

$$
\begin{aligned}
\sum_{i \in J(y)} f_{i}\left[\sum_{j \in J_{i}^{\prime}} f_{j}\right]^{2} & \leqq\left[\sum_{z \in A_{y}} \sum_{i \in J(z)} f_{i}\right]^{3} \\
& \leqq k^{2} \sum_{z \in A_{y}}\left[\sum_{i \in J(z)} f_{i}\right]^{3}
\end{aligned}
$$


thus summing over $y \in Z_{\delta}^{2}$ in (44), we obtain that the constant $K_{3}=4 k^{2}\left(1+\frac{\bar{c}}{c}\right)$ is so large that

$$
\sum_{i \in J} f_{i}\left[\sum_{j \in J_{i}^{\prime}} f_{j}\right]^{2} \leqq K_{3} W\left(\omega_{t}, \mu, \sigma\right),
$$

whence

$$
S_{4} \leqq K_{4} W\left(\omega_{t}, \mu, \sigma\right)
$$

follows by (42) with $K_{4}=1+K_{3}$.

Since each of $Q_{1}, Q_{2}, Q_{31}, Q_{32}, Q_{33}$ is bounded by a linear combination of $S_{1}, S_{2}$, $S_{3}, S_{4}$, our inequalities (22), (25), (37), (46) result in (6) with a universal constant $K$.

\section{A priori Bounds}

First we show that any $g$-tempered solution $\omega_{t}$ satisfies (7), $g$ is an SI-function. Let $T>0$ and consider the unique solution $r(t), 0 \leqq t \leqq T$ of the integral equation

$$
r(t)=\sigma+K \int_{t}^{T} g(|\mu|+r(s))\left[\bar{W}_{g}\left(\omega_{s}\right)\right]^{1 / 2} d s,
$$

then $r$ is differentiable with a bounded derivative $r^{\prime}$, so that (6) implies

$$
\begin{aligned}
& \frac{d}{d t} e^{-K t} W\left(\omega_{t}, \mu, r(t)\right)=-K e^{-K t} W\left(\omega_{t}, \mu, r(t)\right) \\
& \quad+e^{-K t}\left[\frac{\partial}{\partial t} W\left(\omega_{t}, \mu, r(t)\right)+r^{\prime}(t) \frac{\partial}{\partial \sigma} W\left(\omega_{t}, \mu, r(t)\right)\right] \leqq 0
\end{aligned}
$$

for each fixed $\mu$ and $\sigma$ if $0 \leqq t \leqq T$, so that

$$
W\left(\omega_{T}, \mu, \sigma\right) \leqq W\left(\omega_{0}, \mu, r(0)\right) e^{K T}
$$

as $r(T)=\sigma$. Suppose now that $\sigma \geqq g(|\mu|)$, then (47) implies $r(0) \geqq g(|\mu|)$, thus (49) turns into

$$
\bar{W}_{g}\left(\omega_{T}\right) \leqq \bar{W}_{g}\left(\omega_{0}\right) e^{K T}\left[\sup _{\mu} \sup _{\sigma \geqq g(|\mu|)}\left(\frac{r(0)}{\sigma}\right)^{2}\right],
$$

where $r(0)$ and $\sigma$ are related by (47).

Let $z(t)=\int_{0}^{t}\left[\bar{W}_{g}\left(\omega_{s}\right)\right]^{1 / 2} d s$, since $r(0) \geqq r(s) \geqq \sigma \geqq g(|\mu|) \geqq 1$ and $g(u+v) \leqq g(u)+g(v)$ if $u, v$ are positive, we obtain from (47) that

$$
r(0) \leqq \sigma+K(\sigma+g(r(0))) z(T) .
$$

However, $g(u) \leqq 1+2 \sqrt{u}$, thus

$$
r(0) \leqq \sigma+2 K(\sigma+\sqrt{r(0)}) z(T)
$$

whence

$$
\sqrt{r(0)} \leqq \sqrt{\sigma}(1+4 K z(T))
$$


follows directly. Substituting (53) into (51), by the subadditive property of $g$ it follows that

$$
\begin{aligned}
r(0) & \leqq \sigma+K\left(\sigma+g\left(\sigma[1+4 K z(T)]^{2}\right)\right) z(T) \\
& \leqq \sigma+K\left(\sigma+(1+\sigma) g\left([1+4 K z(T)]^{2}\right)\right) z(T) \\
& \leqq \sigma \bar{K}\left(1+z(T) g\left(z^{2}(T)\right)\right),
\end{aligned}
$$

where $\bar{K}>K$ is a new constant; (50) and (54) yield (7), i.e.

$$
z^{\prime}(t) \leqq \sqrt{w} \bar{K} e^{\bar{K} t}\left(1+z g\left(z^{2}\right)\right), \quad t \geqq 0,
$$

provided that $\bar{W}_{g}\left(\omega_{0}\right) \leqq w$. It will be important later that $\bar{K}$ does not depend on $g$.

Introduce now the function $G=G(z), z \geqq 0$ by

$$
G(z)=\int_{0}^{z}\left(1+u g\left(u^{2}\right)\right)^{-1} d u
$$

$G$ is a differentiable and strictly increasing function of $z \geqq 0$ and, by substituting $u^{2}=v$ into (56), we see that $\lim _{u \rightarrow+\infty} G(u)=+\infty$, i.e. $G$ has a continuous inverse function $G^{-1}=G^{-1}(v)$ defined for each $v \geqq 0$. Since any non-negative solution $z=z(t)$ of (55) satisfies

$$
G(z(t)) \leqq \sqrt{w}\left(e^{\bar{K} t}-1\right),
$$

we have

$$
z(t) \leqq G^{-1}\left(\sqrt{w}\left(e^{\bar{K} t}-1\right)\right)<+\infty \text { for each } t \geqq 0 .
$$

The comparison of (55) and (58) yields an explicit bound for $\bar{W}_{g}\left(\omega_{t}\right)$. What we have proven is the basic result of this paper:

Proposition 2. For each SI-function $g$ and $w>0$ there exists a continuous function $\varrho(t)=\varrho_{g}(t, w)$ defined for $t \geqq 0$ such that $\bar{W}_{g}\left(\omega_{t}\right) \leqq \varrho_{g}(t, w)$ for each $t \geqq 0$, whenever $\omega_{t}$ is a g-tempered solution with $\bar{W}_{g}\left(\omega_{0}\right) \leqq w$.

As a consequence we show that the distance travelled by a given particle is also uniformly bounded in finite intervals of time.

Proposition 3. For each SI-function $g$ and $u>0, w>0$ there exists a continuous function $\sigma(t)=\sigma_{g}(t, u, w)$ defined for $t \geqq 0$ such that $\left|x_{i}\left(\omega_{s}\right)-x_{i}\left(\omega_{0}\right)\right| \leqq \sigma_{g}(t, u, w)$ if $0 \leqq s \leqq t,\left|x_{i}\left(\omega_{0}\right)\right| \leqq u, \bar{W}_{g}\left(\omega_{0}\right) \leqq w$ and $\omega_{t}$ is a g-tempered solution.

Proof. We know that $\left|v_{i}\right| \leqq g\left(\left|x_{i}\right|\right) \sqrt{\varrho_{g}(t, w)}$, set

$$
\begin{aligned}
\sigma_{i} & =\sup _{0 \leqq s \leqq t}\left|x_{i}\left(\omega_{s}\right)-x_{i}\left(\omega_{0}\right)\right| \\
\text { and } z & =\int_{0}^{t} \sqrt{\sigma_{g}(s, w)} d s, \text { then } \\
\sigma_{i} & \leqq g\left(u+\sigma_{i}\right) z \leqq 2 g(u) z+2 \sqrt{\sigma_{i}} z
\end{aligned}
$$


follows by the subadditivity of $g$, whence $\left(\sqrt{\sigma_{i}}-z\right)^{2} \leqq(g(u)+z)^{2}$, i.e.

$$
\sigma_{i} \leqq \max \left\{z^{2},(g(u)+2 z)^{2}\right\} \leqq(g(u)+2 z)^{2} .
$$

Substituting (61) into (60) we obtain that

$$
\sigma_{i} \leqq g\left[u+(g(u)+2 z)^{2}\right] z,
$$

which proves the statement.

The use of $W$ instead of $H$ is justified only if we know that

$$
\boldsymbol{\Omega}_{g}=\left\{\omega ; \bar{W}_{g}(\omega)<+\infty\right\}
$$

for each SI-function.

Proposition 4. There exist such constants $B$ and $C>0$ depending only on $U$ that $\bar{H}_{g}(\omega) \leqq \bar{W}_{g}(\omega) \leqq B+C \bar{H}_{g}(\omega)$ if $\omega \in \boldsymbol{\Omega}_{g}$.

Proof. The first inequality is trivial. For the second one let $N_{x}(\omega)$ denote the number of points of $\omega$ in the unit square of center $x \in Z^{2}$. Since $U$ is a superstable pair potential (see Section 1 in [4]) there exist such constants $B_{1}$ and $C_{1}>0$ that

$$
\begin{aligned}
& \sum_{x \in \mathbf{Z}^{2}}\left(C_{1} N_{x}^{2}\left(\omega_{\mu, \sigma}\right)-B_{1} N_{x}\left(\omega_{\mu, \sigma}\right)\right) \\
& \quad \leqq H(\omega, \mu, \sigma)
\end{aligned}
$$

where $\omega_{\mu, \sigma}$ denotes the configuration obtained from $\omega$ by deleting the points outside the disc of center $\mu$ and radius $\sigma$. Hence

$$
\sum_{x \in \mathbf{Z}^{2}} N_{x}^{2}\left(\omega_{\mu, \sigma}\right) \leqq C_{2} H(\omega, \mu, \sigma)+B_{2} \sigma^{2}
$$

follows immediately, $B_{2}$ and $C_{2}>0$ are new constants depending only on $U$. The statement of Proposition 4 follows from (64) by a direct calculation.

The proof of Theorem 2 is based on the following result, it is an extension of Proposition 3 in [3].

Proposition 5. If $\omega_{0} \in \boldsymbol{\Omega}_{g}$ holds for a tempered solution $\omega_{t}$ and $g$ is an SI-function, then $\omega_{t}$ is g-tempered.

Proof. There exists an SI-function $h$ for which $\omega_{t}$ is $h$-tempered; we may assume that $g(u) \leqq 2 h(u)$ because $h$ can be replaced by $\frac{1}{2}(g+h)$ otherwise. Let $g_{n}(u)=g(u)$ if $0 \leqq u \leqq n$ and $g_{n}(u)=g(n)+\min \left\{g^{\prime}(n+0) h(u-n), 2(\sqrt{u}-\sqrt{n})\right\}$ if $u \geqq n, n=1,2, \ldots$; it is easy to check that $g_{n}$ is an SI-function and $g(u) \leqq 2 g_{n}(u)$ for each $u$ and $n$; thus $\bar{W}_{g_{n}}\left(\omega_{0}\right) \leqq 4 \bar{W}_{g}\left(\omega_{0}\right)=w$. On the other hand, $g_{n}$ has the same asymptotic order at infinity as $h$ does, therefore $\omega_{t}$ is $g_{n}$-tempered for each $n$ and Proposition 2 implies

$$
\sigma^{-2} W\left(\omega_{t}, \mu, \sigma\right) \leqq \varrho_{g_{n}}(t, w) ;
$$

at least if $\sigma \geqq g_{n}(|\mu|)$. However, $\lim \varrho_{g_{n}}(t, w)=\varrho_{g}(t, w)$ uniformly in finite intervals of time as $g_{n}(u)=g(u)$ if $n>u$ [see $\left(5^{n}\right)$ and (58)]; thus the statement follows directly from (65) and Proposition 4. 
Finally we deduce a contraction property of the equations of motion from the $g$ Lipschitz condition. Let $\omega$ and $\bar{\omega}$ belong to $\overline{\boldsymbol{\Omega}}$, for convenience we assume that $J(\omega)=J(\bar{\omega})=J$. Further, $\bar{x}_{i}=x_{i}(\bar{\omega}), \bar{v}_{i}=v_{i}(\bar{\omega}), w>0, p>0, g$ is an SI-function, $L$ and $f$ are defined in (1) and (i), (ii), (iii), $R$ is the range of $U$ in the forthcoming notation.

$$
\begin{gathered}
\lambda_{n}(w)=\left[\sqrt{w} g(4 R n+4 R) L\left(w g^{2}(4 R n+4 R)\right)\right]^{1 / 2}, \\
d_{n}(\omega, \bar{\omega} ; w) \\
=\sum_{i \in J} f\left(4 R n-\left|x_{i}\right|\right) f\left(4 R n-\left|\bar{x}_{i}\right|\right)\left(\lambda_{n}(w)\left|x_{i}-\bar{x}_{i}\right|+\left|v_{i}-\bar{v}_{i}\right|\right), \\
M_{1}(w)=1, M_{n}(w)=\lambda_{1}(w) \lambda_{2}(w) \ldots \lambda_{n-1}(w) \text { if } n>1, \text { and } \\
D_{p}(\omega, \bar{\omega} ; w)=\sum_{n=1}^{\infty} \frac{p^{n}}{n !} M_{n}(w) d_{n}(\omega, \bar{\omega} ; w) .
\end{gathered}
$$

It is easy to check that $(\mathrm{U})$ implies that $\lambda_{n}(w)=o(n)$, i.e. $\lim _{n} \frac{1}{n} \lambda_{n}(w)=0$ for $w>0$, thus $D_{p}<+\infty$ if $\omega, \bar{\omega}$ are from $\overline{\boldsymbol{\Omega}}$. Further, $D_{p}(\omega, \bar{\omega} ; w)>0$ if $\omega \neq \bar{\omega}$, and for a sequence $\omega_{n} \in \boldsymbol{\Omega}_{g}^{h}$ we have $\lim \omega_{n}=\omega$ if and only if there exist such $p>0$ and $w>0$ that $\lim _{n} D_{p}\left(\omega_{n}, \omega ; w\right)=0$.

Proposition 6. Suppose that $U$ satisfies a g-Lipschitz condition with an SI-function $g$, then there exists a constant $q$ depending only on $U$ such that $D_{1}\left(\omega_{t}, \bar{\omega}_{t} ; w\right)$ $\leqq D_{1+q t}\left(\omega_{0}, \bar{\omega}_{0} ; w\right)$, if the solutions $\omega_{s}, \bar{\omega}_{s}$ satisfy $\bar{W}_{g}\left(\omega_{s}\right) \leqq w, \bar{W}_{g}\left(\bar{\omega}_{s}\right) \leqq w$ for $0 \leqq s \leqq t$, and $J\left(\omega_{0}\right)=J\left(\bar{\omega}_{0}\right)$.

Proof. It is essentially the same as that of Proposition 4 in [3]. If $\max \left\{\left|x_{i}\right|,\left|\bar{x}_{i}\right|\right\}$ $\leqq(4 n+3) R$, then from (U) and (11) we obtain that

$$
\begin{aligned}
& \sum_{j \in J_{i}^{\prime}}\left|\operatorname{grad} U\left(x_{i}-x_{j}\right)-\operatorname{grad} U\left(\bar{x}_{i}-\bar{x}_{j}\right)\right| \\
& \leqq L\left(w g^{2}(4 R n+4 R)\right)\left[A \sqrt{w} g(4 R n+4 R)\left|x_{i}-\bar{x}_{i}\right|+\sum_{j \in J_{i}^{\prime}}\left|x_{j}-\bar{x}_{j}\right|\right]
\end{aligned}
$$

$\frac{d}{d t}\left|x_{i}-\bar{x}_{i}\right| \leqq\left|v_{i}-\bar{v}_{i}\right|$ almost everywhere, the cardinality of $J_{i}^{\prime}$ and the velocities appearing in the derivative of $f$ can also be estimated by $A \sqrt{w} g(4 R n+4 R)$, see (10), (11). Further, $d_{n}\left(\omega_{t}, \bar{\omega}_{t} ; w\right)$ is an absolutely continuous function of $t$, thus taking into account the properties of $f$ and the above relations, we obtain that

$$
\frac{d}{d s} d_{n}\left(\omega_{s}, \bar{\omega}_{s} ; w\right) \leqq q \lambda_{n}(w) d_{n+1}\left(\omega_{s}, \bar{\omega}_{s} ; w\right)
$$

a.e. in $(0, t)$. Hence, following the proof of Proposition 4 in [3], we obtain the statement of Proposition 6.

The tools needed to prove the results formulated in Section 3 are all summarized in the propositions of this section. 


\section{Proof of the Theorems}

Theorems 1-3 follow from Propositions 2-6 by standard methods discussed in details in [3], this is why here we only sketch them.

For the proof of Theorem 1, consider the sequence $\varphi_{n}(t, \omega)$ of approximate solutions. In view of Proposition $4, \bar{W}_{g}(\omega)<+\infty$ with an SI-function $g$, thus $\bar{W}_{g}$ is uniformly bounded at $t=0$ for the corresponding finite systems. Therefore Propositions 2 and 3 imply that, given $i$ and $T,\left|v_{i}\left(\varphi_{n}(t, \omega)\right)\right| \leqq \sqrt{\varrho}$, where $\varrho$ does not depend on $n$ and $t \leqq T$; so that using the diagonal method, we can select a convergent subsequence $\varphi_{n_{k}}(t, \omega)$. Because interparticle distances are bounded away from zero if $\bar{W}_{g}$ is bounded, this limit is necessarily a solution.

The first statement of Theorem 2 is just Proposition 5. For the second one it is enough to remark that $\bar{\omega}_{t}=\omega_{s-t}^{+}$is a $g$-tempered solution if $\omega_{s} \in \boldsymbol{\Omega}_{g}, s>0$, as follows from Proposition 5. Thus $\omega_{0}=\bar{\omega}_{s}^{+} \in \boldsymbol{\Omega}_{g}$ and $\omega_{t}$ is $g$-tempered.

Theorem 3 is a direct consequence of Propositions 2, 4, and 6.

\section{References}

1. Lanford,O.E.III.: The classical mechanics of one-dimensional systems of infinitely many particles. Commun. math. Phys. 9, 169-191 (1968)

2. Lanford,O.E.III.: Time evolution of large classical systems. In: Dynamical systems, theory, and applications. Lecture notes in physics, Vol. 38, pp. 1-111. Berlin-Heidelberg-New York: Springer 1975

3. Dobrushin, R.L., Fritz, J.: Non-equilibrium dynamics of one-dimensional infinite particle systems with a hard-core interaction. Commun. math. Phys. 55, 275-292 (1977)

4. Ruelle, D. : Superstable interactions in classical statistical mechanics. Commun. math. Phys. 18, 127159 (1970)

5. Ruelle,D. : Classical statistical mechanics of a system of particles. Helv. Phys. Acta 36, 183-197 (1963)

6. Dobrushin, R.L.: Conditions on the asymptotic existence of the configuration integral for Gibbs distributions. (In Russian) Teor. Ver. Prim. 9, 626-643 (1964)

7. Ruelle,D.: Statistical mechanics, rigorous results. New York-Amsterdam: Benjamin 1969

Communicated by J. L. Lebowitz

Received July 13, 1977 
